\title{
ЛЭМБОВСКИЙ СДВИГ дЛЯ ВОДОРОДА И ДЕЙТЕРИЯ *)
}

\author{
E. E. Caлnemep
}

\section{Om переводиика}

Настоящий об̆зор Салпетера посвящён лэмбовскому сдвигу для водорода и дейтерия - пробному камню одной из интереснейших проблем современной теоретической и экспериментальной физики - радиационным поправкам в квантовой электродинамике **).

До 1947 г. никакой проблемы фактически не существовало. Спектр атома водорода тогда теоретически описывался уравнением Дирака для одного электрона во внешнем поле. Многочисленные әкспериментальные исследования, проводившиеся оптическими методами, в пределах ошибок опыта хорошо подтверждали выводы теории. В частности, подтверждалось предсказадное теоретически совпадение $2 S_{1 / g}$ и $2 P_{\frac{1}{2}}$ уровней. Эксперименты некоторых авторов, правда, указывали на возможность небольшого расщепления этих уровней, но эти результаты не были убедительны, так как получавшееся расщепление имело порядок вероятной ошибки измерения.

Исключительно большая точность оптических методов $\left(\sim 10^{-6}\right.$ по отношению к основному терму), казалось, ставила предел скольконибудь существеныым новым эксперименталыным успехам в этом направлении.

Однако применеңие радиочастотной техники позволило повысить точность измерений на много порядков. Лежавший на пределе возможностей оптических методов сдвиг $2 P_{1 / 2}$ и $2 S_{1 / 2}$ уровней в 1947 г. был с достоверностью обнаружен Лэмбом и Ризерфордом, а в 1952 г. измерен с точностью до $10^{-10}$ по отношению ко основному терму атома водорода. Насколько нам известно, такая точность (десять знаков!) не была еще достигнута вообще ни в одном физическом измерении.

Успехи экспериментальной техники стимулировали развитие теории. Если исходить из строгих уравнений квантовой электродинамики для атома водорода, то оказывается, что уравнение Дирака для частицы в кулоновском поле получается лишь как первое неисчезающее приближение теории возмущений. Следующие приближения должны были давать

*) Phys. Rev. 89, 92 (1953), перевод Ю. М. Широкова.

* Дополительный список литературы на русском языке по этим вопросам см. в конце статьи. 
малые поправки, названые впоследствин радиационными. Однако вследствие внутренней противорецивости квантовой электродинамики (и по сей день не являюшейся логически замкнутой теорией) высшие приближения при расиёте давали не малые поправки, а бесконечные, расходяпиеся члены.

До 1947 г. эти расходящиеся выражения обычно просто отбрасывались как бессмысленные, тем более, что первое неисчезающее приближение во всех случаях давало достаточное совпадение с экспериментом. Лишь одна из радиационыых поправок, называемая поляризацией вакуума, была вычислена в довоенные годы. Однако объяснить лэмбовский сдвиг sа счёт поляризации вакуума не удалось, так как соответствующая поправка была слишком мала по величине и к тому же имела противоположньй знак.

С 1947 г. различные авторы начали разрабатывать методы выделения малых поправок из бесконечных выражений для высших приближений в квантовой электродинамике. Главная трудность этой задади заключалась в том, что операция разделения бесконечного выражения на конечную и бесконечную части, вообе говоря, неоднозначна. Эту трудность удалось преодолеть с помошью прнведения теории возмущений к релятивистски инвариантному виду. Оказалось, что релятивистски инвариантное выделение конечных радиационных поправок может быть проведено однозначным образом в любом приближении. При учёте радиационных поправок в. проблеме атома водорода величина ләмбовского сдвига оказывается конечной и преграсно согласуюшейся с экспериментальными даными.

Кроме радиационных поправок на величину лэмбовскиго сдвига влияет целый ряд других факторов, что подробно разбирается в приводимой ниже статье. При этом оказывается, что с угётом всєх известных ґоправок теория $\mathrm{c}$ точностью до одного мегацикла (9 знаков по отношению к основному атомному терму!) совпадает с опытом.

Тем интереснее, что при дальнейшем повышении точности появляется определённое расхождение теории с опытом: Это расхождение оказалось равным 0,5 жәи при точности 0,1 жақ. Для объяснения этого расхождения, новидимому, нужны какие-то новые физические идеп. Представляотся желательными дальнейшие экспериментальные исследования в этой области, так как достигнутая точность измерений, повндимому, не является предельной.

\section{§ 1. ВВЕДЕНИЕ}

За последние годы значительно увеличилась точность экспериментального определения лэмбовского сдвига. Подробное описание экспериментальной техники и расчётов по прецизионному измерению лэмбовского сдвига содержится в серии статей "Тонкая структура атома водорода" ${ }^{1-3}$. Полным лэмбовским сдвигом (обозначен через $S$ и выражен в $\left.M 2 u_{4}\right)$ насывается разность энергий между $n^{2} S_{1 / 2}$ и $n^{2} P_{1 / 2}$ уровнями водородоподобного атома. В элементарной теории Дирака для электрона в кулоновском поле эти уровни совпадают. в. настоящей статье мы будем иметь дело главным образом со случаем $n=2$ для водорода и дейтерия. Для этого случая $S$ определено экспериментально с точностью до одной десятой $M$ ми. Различными авторами было рассчитано влияние большого числа эффектов на лэмбовский сдвиг, Главная цель настоящей статьи - собрать 
воедино результаты предыдущих вычислений и рассчитать влияние ещё нескольких членов. Поправки к лэмбовскому сдвигу относительных порядков $a^{3},\left(\frac{a m}{M}\right),\left(\frac{a^{-1} m^{2}}{\bar{M}^{2}}\right)$ и выше в настоящей статье рассматриваться не будут.

Смещение энергетических уровней связанного электрона в кулоновском поле выражается в виде ряда по степеням постоянной тонкой структуры $\alpha$. Результаты проделанных ранее вычислений для всех членов двух низших порядков по $\alpha$ собраны в $\$ 2$. Это смецение в кулоновском поле ответственно за главную часть лэмбовского сдвига. Учёт конечной массы и внутренней структуры атомных ядер приводит к возникновению поправок к этому смещению. Некоторые поправки, обусловленные конечной массой атомного ядра, были вычислены ранее. Остальные поправки в низшем порядке по $a$, зависящие от конечной массы ядра, обсуждаются в § 3 . Для дейтерия были получены дополнительные поправки за счёт учёта конечных размеров ядра. Эти поправки приведены в $§ 4 . \mathrm{B}$ \$ 5 разбирается вопрос о влиянии внутренней структуры отделыых нуклеонов. В $\$ 6$ ре. зультаты этих вычислений сравниваются с последними экспериментальными данными по лэмбовскому сдвигу. В приложении даётся сводная таблица всех обсуждаемых в статье поправок к лэмбовскому сдвигу.

\section{§ 2. ЛЭМБОВСКИЙ СДВИГ \\ В ЗАДАННОМ КУЛОНОВСКОМ ПОЛЕ}

В этом параграфе собраны все результаты произведённых различными авторами вычислений сдвига уровня в заданном кулоновском поле, т. е. для атома с бесконечно тяжёлым точечным ядром.

В этих вычислениях встречаются два типа разложений. Последующие приближения по виртуальному полю излучения дают разложение по степеням $\alpha$. Последующие приближения по кулоновскому полю дают разложение по степеням $Z \alpha$, где $Z$ - атомный номер (в нашем случае единица). Для главного квантового числа $n=2$ и для заряда $Z=1$ результат в низшем порядке как по $\alpha$, так и по $Z x$ имеет вид:

$$
\begin{aligned}
S_{\infty}^{(1)}=\left(\frac{\alpha^{3} R y_{\infty}}{3 \pi}\right)\left\{\left[\ln \frac{m c^{2}}{R_{0}(2,0)}-\ln 2+\frac{5}{6}\right]-\right. \\
\left.-\frac{1}{5}-\left[\ln \frac{\mathrm{Ry}_{\infty}}{k_{0}}(2,1)-\frac{3}{8}\right]\right\},
\end{aligned}
$$

где $R y_{\infty}$ - ридберговская постоянная для ядра с бесконечной массой, $m$-масса электрона, $k_{0}(2,0)$ и $k_{0}(2,1)$ - «средние энергии возбуждения", определённые и вычисленные в “. В (1) первый. член в квадратных скобках даёт сдвиг уровня $2 S_{1 / 2}$ без учёта поляризащии 
вакуума. $-\frac{1}{5}$ представляет собой поправку к сдвигу $2 S_{1 / g}$ уровня за счёт поляризации вакуума. Последний член в квадратных скобках описывает сдвиг $2 P_{1 / 2}$ уровня.

При получении уравнения (1) части, соответствующие виртуальным фотонам "низких" и "высоких" импульсов, вычисляются различными способами. Часть, соответствующая низким импульсам, вычисляется нерелятивистским методом Бете ${ }^{5} \mathfrak{c}$ использованием точных волновых функций атома. Эти вычисления дают"

$$
k_{0}(2,0)=(16,646 \pm 0,007) \mathrm{Ry}_{\infty}
$$

и

$$
k_{0}(2,1)=(0,9704 \pm 0,0002) R y_{\infty} .
$$

Для части, соответствующей высоким импульсам, электрон трактуется релятивистски, причём в промежуточных состояниях применяется приближение плоских волн. Эти релятивистские вычисления, приводящие к уравнению (1), были выполнены различными методами ${ }^{6,} 7$.

Наиболее точное значение постоянной тонкой структуры может быть получено посредством измерения тонкой ${ }^{8}$ и сверхтонкой структуры. Ввиду того, что в настоящее время теоретическое истолкование измерений постоянной тонкой структуры ${ }^{9}$ не является строго определённым, мы запишем:

$$
\frac{1}{\alpha}=137,0360+\dot{\varepsilon} .
$$

Поправка $\varepsilon_{\alpha}$ не превышает 0,002 .

Пользуясь величиной $299790,9 \pm 1,0 \kappa \mu / c е к$ для скорости света ${ }^{10}$, значение лэмбовской постоянной $L$ в единицах частоты можно записать в виде

$$
L \equiv\left(\frac{\alpha^{3}}{3 \pi}\right) \mathrm{Ry}_{\infty} c=\left(135,6431-3 \varepsilon_{\alpha} \pm 0,0005\right) \text { Max. }
$$

Величина члена в фигурных скобках в (1) равна ${ }^{4} 7,7567 \pm 0,0005$. Для лэмбовского сдвига в низшем поридке это даёт:

$$
S_{\infty}^{(1)}=\left(1052,14-22 \varepsilon_{\alpha} \pm 0,07\right) \mathrm{Maz} \text {. }
$$

$S(1)$ содержит поправку $-27,13 M 2$ жа счёт поляризации вакуума и $\frac{1}{2} L=+67,82 \mathrm{Mzu}$ за счёт аномального магнитного момента электрона.

Следующий член разложения по степеням $Z \alpha$ (кулоновский потенциал, действующий дважды, и т. д.) был вычислен в ${ }^{11}$. Этот 
थлен с учётом поляризации вакуума равен

$$
S^{(2, Z)}=+7,14 \quad M 2 u \text {. }
$$

Следующий член разложения по степеням $\alpha$ (два виртуальных фотона) состоит из трёх частей: а) поправка четвёртого порядка к $S$ за счёт аномального магнитного момента электрона ${ }^{12}$ равна - $0,94 M_{2}$, б) поправка четвёртого порядка за счёт поляризации вакуума ${ }^{13}$ равна $-0,24$ Мгы и в) прочие радиационные поправки четвёртого порядка ${ }^{14}$ равны $(-0,24 \pm 0,10)$ Mzц. Сумма всех этих членов порядка $a S^{(1)}$ и $Z \alpha \cdot S^{(1)}$ равна

$$
S^{(2)}=(6,20 \pm 0,10) M 2 \not \text {. }
$$

Члены относительных порядков $\alpha^{2}, Z x^{2}, Z^{2} \alpha^{2}$ и выше до сих nор не были вычислены. Так как член $S^{(2, Z)}$ относительного порядка $Z \alpha$ численно гораздо больше, чем можно было ожидать, то не исключено, что член порядка $Z^{2} \alpha^{2}$ будет иметь заметную величину. Мы обозначим поправку за счёт этого члена к лэмбовскому сдвигу через $S_{Z}^{(3)}$; поправку за счёт остальных до сих пор ещё не вычисленных членов обозначим через $S_{R^{*}}$. Окончательно численное значение лэмбовского сдвига в заданном кулоновском поле ( $n=2$, $Z=1)$ равно

$$
S_{\infty}=\left(1058,34-22 \varepsilon_{\alpha}+S_{Z}^{(3)}+S_{R} \pm 0,12\right) M 2 \text { w. }
$$

\section{§ З. ПОПРАВКИ ЗА СЧЁТ КОНЕЧНОЙ МАССЫ ЯДРА}

$\mathrm{B}^{15}$ были вычислены поправки порядка $\alpha\left(\frac{m}{M}\right)$ к тонкой структуре водорода за счёт конечной массы $M$ протона. Эти члены связаны с фейнмановскими диаграммами (например, обмен двумя виртуальными фотонами мәжду электроном и протоном), существенно отличными от диаграмм, относяцихся к собственно лэмбовскому сдвигу и эффекту поляризации вакуума. Для уровней $2 S_{1 / 2}$ и $2 P_{1 / 2}$ эти члены различны. Поэтому они дают поправку к лэмбовскому сдвигу порядка $\left(\begin{array}{c}m \\ M\end{array}\right) S$, что подтверждается экспериментально. Для водорода эти члены дают сдвиг уровней $+0,379$ Mzъ в $2 S_{1 ;}$ состоянии и $-0,017$ M2ע в $2 P_{1 / 2}$ состоянии. Таким образом, соответствуюцая поправка к лэмбовскому сдвигу равна + 0,396. M2z. Если пренебречь внутренней структурой ядра, то для дейтерия эти члены вдвое меныше, чем для водорода. Пренебрежение структурой дейтерона даёт для этих членов ошибку порядка $10 \%$.

Теперь мы должны добавить поправки за счёт приведённой мaссы в выражение (6) с тем, чтобы учесть конечную массу атомного. 
ядра. Мы подставим эти поправки только в главный член $S^{(1)}$, определяемый уравнением (4).

Как показано в ${ }^{15}$, Эти поправки в принципе могут быть получены путём вычисления лэмбовского сдвига в целом при помощи четырёхмерного ковариантного волнового уравнения для системы двух тел в связанном состоянии. Этот метод подобен применённому в работе $^{7}$ для вычисления ләмбовского сдвига, но сложнее его. В обоих методах содержится, например, оператор, определяемый уравнением (12) из работы ${ }^{7}$. В методе, использованном в ${ }^{7}$, среднее от этого оператора вычисляется при помощи обычных трёхмерных волновых функций и употребляются только матричные элементы переходов между состояниями, удовлетворяющими уравнению Дирака (в низшем порядке по $Z \alpha$ ). В другом методе среднее от этого оператора надо вычислять при помощи четырёхмерных волновых функций системы двух тел и при этом уже требуются матричные элементы переходов между состояниями, не удовлетворяющими уравнению Дирака.

Но для лэмбовского сдвига в низшем порядке по $Z \alpha$ ядро взаимодействует с электроном лишь однажды, так что в выражении для сдвига не встречаются знаменатели, содержащие энергию ядра. Масса ядра входит только в простом виде, и следовательно, эффект, вносимый конечной массой ядра, может быть легко получен путём анализа обычных вычислений для̆ электрона в заданном кулоновском потенциале.

Сначала мы рассмотрим проведённое в $^{5}$ нерелятивистское вычисление для части ләмбовского сдвига, соответствующей низким импульсам. Пусть $M$ - масса ядра, $m$-масса электрона и $\mu$-приведённая масса электрона. Перейдём к системе покоящегося центра инерции. В этой системе импульс электрона $\mathbf{p}_{e}$ и импульс ядра $\mathbf{p}_{N}$ равны по величине и противоположны по направлению. После исключения движения центра инерции уравнение Шредингера содержит приведённую массу $\mu$ и «относительный импульс» $\mathbf{p}$. Тогда $\mathbf{p}=\mu \mathbf{v}$, где $\mathbf{v}$-относительная скорость. $\mathbf{p}_{e}$ равно произведению $m$ на «абсолютную скорость электрона»

$$
\left(\frac{M}{m}+M\right) \mathbf{v}
$$

Таким образом, рточно равно $\mathbf{p}_{e}$.

$\mathrm{B}^{5}$ дираковская матрица $\alpha$ заменена скоростью электрона, которая равна $\frac{\mathbf{p}_{e}}{m}$. Полученное выражение пропорционально (см. уравнение (5) в $^{5}$ )

$$
\frac{1}{m^{2}} \int d k \sum_{n} \frac{\left.|<0| \mathbf{p}_{e}|n\rangle\right|^{2}\left(E_{n}-E_{0}\right)}{\left(E_{n}-E_{0}+k\right)},
$$

где $k$ - энергия виртуального фотона, $E_{0}$ - собственное значение 
энергии уравнения Шредингера (содержащего приведённую массу $\mu$ и отнюсительные координаты и импульсы) для рассматриваемого состояния; $E_{n}-$ энергия любого другого состояния.

Поскольку $\mathbf{p}_{e}$ равно относительному импульсу $\mathbf{p}$, все величины в интеграле $(7)$, за исключением множителя $m^{-1}$, относятся к уравнению ІШредингера, содержащему приведённую массу; $\mu$. Этот интеграл точно равен эквивалентному интегралу для частицы массы $⿻$ в заданном кулоновском поле. Численная величина этого интеграла содержит множитель $\mu^{3}$ (этот множитель получается из квадрата атомной волновой функции). Следовательно, мы должны главную (нерелятивистскую) часть $S_{\infty}^{(1)}$ умножить на $\mu^{3}$ :

$$
\left(\frac{\mu}{m}\right)^{3} \approx\left(1-\frac{3 m}{M}\right)
$$

Величины $\mathrm{Ry}, k_{0}(2,0)$ и $\mathfrak{k}_{0}(2,1)$ в фигурных скобках в (1) получены из нерелятивистского рассмотрения и пропорциональны первой степени массы. Следовательно, эти члены надо умножить на $\left(\frac{i}{m}\right)$, т. е. надо брать численную величину Ry для приведённой массы $\mu$. Остальные члены в фигурных скобках (1), включая и $m c^{2}$, получаются при вычислении части, соответствующей высоким импульсам.

В 7 для вычисления этих членов 4-потенциал sъра разлагается в интеграл Фурье $A_{\boldsymbol{v}}(\mathbf{q})$, где $\mathbf{q}$ - изменение импульса. Далее вычисляются радиационные поправки к рассеянию электрона в этом потенцилле (см. уравнение $(24)$ в ${ }^{7}$ ).

Учёт конечной массы ядра приводит к тому, что форма $A_{4}(\mathfrak{q})$ слегка изменяется п появляется небольшая поперечная компонента $\mathrm{A}(\mathbf{q})$. Уравнение $(24)$ в $^{7}$ содержит истинную массу электрона, которая не зависит от формы потенциала, а следовательно, и от массы ядрэ. Для заданного значения $q$ отношение потенциала, создающего радиационную поправқу, к $A$, $(\mathbf{q})$ пропорционально $q^{2}$ и не зависит от процентного содержания поперечной компоненты для всех членоз, кроме тех, которые обусловлены аномальным магнитным моментом. Тогда зависимость волновзй функции атома и $A_{4}(\mathfrak{q})$ от массы. ядра приводит к тому, что выражение для лэмбовского сдвига для бесконечной массы ядра должно быть умножено на выражение (8). Далее, множитель $m c^{3}$ под логарифмом в фигурных скобках (1) следует из уравнения (24) в ${ }^{7}$ и содержит истинную массу. электрона, тогда как множитель $k_{0}(2,0)$ содержит приведённую массу $\mu$, как указывалось выше. Следовательно, мы должны добавить в. фигурные скобки член

$$
\ln \left(\frac{m}{\mu}\right) \approx\left(+\frac{m}{M}\right)
$$


С другой стороны, поправка $\frac{1}{2} L$ к $S^{(1)}$ за счёт аномального магнитного момента электрона каким-то образом зависит от малой

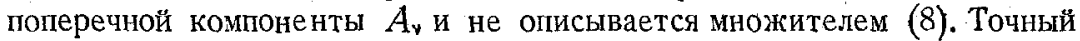
множитель к этому члену ещё не вычислен и мы запишем его в виде ${ }^{16}$

$$
\left[1-\left(1+\varepsilon_{\mu}\right) \frac{m}{M}\right]
$$

где $\varepsilon_{\mu}$ лежит в интервале \pm 2 . Тогда полная поправка к $S^{(1)}$ за счёт приведённой массы в низшем порядке по $\frac{m}{M}$ равна:

$$
\left(\frac{m}{M}\right)\left[-3 S^{(1)}+\left(2-\frac{1}{2} \varepsilon_{\mu}\right) L\right]
$$

Поправки к пэмбовскому сдвигу четвёртого порядка за счёт приведённой массы $S^{(2)}$ до сих пор не вычислены; они не должны превышать $0,030 \mathrm{M} 2$, для водорода и 0,015 Mzш для дейтерия. Поправки за счёт диаграмм, в которых не электрон, а ддро, испускает и поглощает виртуальный фотон, имегот порядок $\left(\begin{array}{c}m \\ M\end{array}\right)^{2} S$ и, следовательно, пренебрежимо малы, Складывая члены, вычисленные в ${ }^{15}$, с выражением (10), получаем полную поправку к $S$ за счёт конечной массы ядра соответственно для водорода и дейтерия:

$$
\begin{aligned}
& \Delta S_{\mathrm{H}}=-\left[1,175+0,037 \varepsilon_{\mu} \pm 0,030\right] \text { Mzu, } \\
& \Delta S_{\mathrm{D}}=-\left[0,588+0,019 \varepsilon_{\mu}=0,030\right] \text { Mzu. }
\end{aligned}
$$

\section{§4. УЧЁТ КОНЕЧНОГО РАДИУСА ДЕЙТЕРОНА}

Рассмотрим влияние конечных размеров дейтерона на электростатическую нерелятивистскую потенциальную энергию атомных состояний дейтерия. Сначала мы вычислим этот эффект, предположив, что атомный электрон вращается вокруг центра инерции дейтерона, а в дальнейшем дадим обоснование этому предположению. Пусть $r$ - расстояние от прэтона до центра инерции дейтерона $(2 r-$ относительное расстокние) и $\gamma^{-1}$ - радиус дейтерона, равный

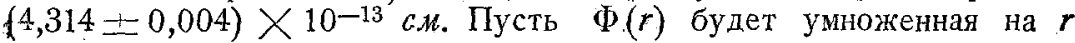
волновая функция дейтерона, нормированная так, что её асимптотическое значение равно $u(r)=e^{-2 \gamma r}$. Пусть $\Delta V(r)-$ разность между электростатической потенциальной энергией электрона на расстоянии $\boldsymbol{r}$ от центра распределения заряда в дейтероне и соответствующей энергией для точечного заряда ядра. Эта поправка к 
потенциалу равна

$$
\left.\begin{array}{rl}
\Delta V(r) & =+e^{2} N \int_{r}^{\infty} d y \Phi^{2}(y)\left[r^{-1}-y^{-1}\right] \\
N^{-1} & =\int_{0}^{\infty} d r \Phi^{2}(r) .
\end{array}\right\}
$$

Так как $\Delta V(r)$ отлично от нуля лишь для зиачений $r$, значительно меньших размеров атома, то при вычислении среднего значения $<\Delta V\rangle$ атомную волновую функцию $\psi(r)$ можно заменить на её значение в начале координат $\psi(0)$ :

$$
\left.\begin{array}{l}
<\Delta V>=|\Psi(0)|^{2} \int d^{3} r J V(r)=\left(2 \pi \frac{e^{2}}{3}\right)|\Psi(0)|^{2}<r^{2}> \\
<r^{2}>=N \int_{0}^{\infty} d r r^{2} \Phi^{2}(r) .
\end{array}\right\}
$$

Уравнение (13) и приближённое значение для $\left\langle r^{2}\right\rangle$ было впервые получено в ${ }^{11}$. Нормировочный множитель $N$ равен $4 \gamma\left(1-\gamma r_{0}\right)^{-1}$, где $r_{0 t}$ - эффективный размер триплетного $n$ - $p$-потенциала. $r_{0 t}$ может быть получено ${ }^{17}$ из экспериментов по рассеянию при малых энергиях и слабо зависит от выбора формы потенциальной ямы. Интеграл в (13) равен

$$
F N \int_{0}^{\infty} d r r^{2} u^{2}(r)=\frac{F}{8 \gamma^{2}} .
$$

Множитель $F$ блхзок к единице, так как максимум подинтегрального выражения лежит вне радиуса действия ядерных сил, где $F$ практически равно своему асимптотическому значению $u$. Произведение $F\left(1-\gamma r_{0 t}\right)^{-1}$ слабо зависит от формы потенциальной кривой и равно примерно 1,60 для прямоугольной ямы и 1,62 для юкавского потен пиала. Мы примем значение $(1,61 \pm 0,05)$. Ошибка происходит от неопределённости в значении $r_{0 t}$ и в форме потенциальной кривой. Так как $\langle\Delta V\rangle$ в (13) равно нуло для $2 P$-состояния и не равно нулю для $2 S$-состояния, то отсюда возникает доступная экспериментальному наблюдению поправка к лэмбовскому сдвигу для дейтерия, равная

$$
\langle\Delta V\rangle=+(0,733 \pm 0,025) M 2 u \text {. }
$$

мы должны теперь оправдать сделанное при выводе (14) допущение о том, что электрон рассматривается как вращающийся вокруг центра инерции дейтерона, а не вокруг протона. Рассмотрим задачу 
в импульсном пространстве. Пусть $\varphi(p)$ - атомная волновая функция в импульсном пространстве, т. е. компонента Фурье от $\psi(\mathbf{r})$, и $a_{0}$ боровский атомный радиус. Тогда $c$ возрастанием $p$ при $\mathbf{p} a_{0} \gg h$ $\varphi(\mathbf{p})$ быстро спадает приблизительно как $p^{-4}$. Компонента Фурье для кулоновского потенциала пропорциональна $\mathbf{q}^{-2}$, где $\mathbf{q}-$ изменение импульса. Компонента Фурье $\Delta V(\mathbf{q})$ от $V(\mathbf{r})$ пропорциональна функции порядка $\left(q^{2}+h^{2} \gamma^{2}\right)^{-1}$, т. е. практически постоянна вплоть до импульсов порядка' $h \gamma$ или около 50 Мәв/с. Приближение, в котором: электрон считается вращающйся вокруг центра инерции дейтерона, является достаточно точным для импульсов $p$, таких, что соответствующая кинетическая энергия электрона пренебрежимо мала по сравненйо с энергией связи дейтерона, т. е. $p \leqslant 2$ Mэв/c. Выражение для $\langle\Delta V\rangle$ в импульсном пространстве запишется в виде:

$$
\iint d^{3} p d^{3} q \varphi^{*}(\mathbf{p}) \Delta V(\mathbf{q}) \varphi(\mathbf{p}+\mathbf{q})
$$

Так как $p^{2} \varphi(p)$ убывает как $p^{-2}$ при $p \gg \frac{h}{a_{0}} \sim 5$ кэв, то величина интеграла (15) зависит главным образом от $p, q \leqslant \frac{h}{a_{0}} \leqslant 2 M э в / c$. Тогда ошибка, вносимая нашим предположением о щентре вращения әлектрона, будет иметь относительный порядок всего лишь $\frac{h c}{a_{0}} 2 M_{\ni}$ или менее $1 \%$. В уравнении (15) $\Delta V(\mathbf{q})$ может быть заменено его значением при $q=0$, что приведёт точно к уравнению (13). Таким образом, в настоящем случае результат существенно иной, чем для учёта влияния конечного радиуса дейтерона на сверхтонкую структуру ${ }^{18}$. В последнем случае $\Delta V(\mathbf{q})$ заменяется в озр аста пощ ей функцией и величина поправки к соответствующему интегралу зависит главным образом от боль пи х значений $q$, на которые наше предположение о вращении не распространяется.

Мы вычислили лишь среднее значение поправочного потенциала $\Delta V(r)$ и пренебрегли радиационными поправками к нему. Пользуясь уравнением (24) из ${ }^{7}$ в импгульсном пространстве, можно показать, что эти радиационные поправки меньше самого́ среднего значения в $\alpha^{2} \ln \alpha$ раз, и следовательно, ими можно пренебречь. Как показано $\mathrm{B}^{15}$, влияние структуры дейтерона на поправки за счёт конечной массы к тонкой структуре также мало. Пренебрежение этими эффектами даёт дополнительную ошибку порядка $\pm 0,02 \mathrm{Mzи}$ для лэмбовского сдвига в дейтерии, приведённого в $\$ 3$.

\section{§ 5. УЧЁТ ВНУТРЕННЕЙ СТРУКТУРЫ НУКЛЕОНА}

До сих пор мы предполагали, что нейтроны и протоны представляют собой точечные частицы, подчиняющиеся статистике фермиДирака и лишённые какой бы то ни было внутренней структуры. В действительности нуклеоны должны вести себя йначе, так как 
они сильно взаимодействуют с виртуальным мезонным полем. Это взаимодействие, повидимому; даёт: а) увеличение аномального магнитного момента, б) размазывание электрического заряда и магнитного мсмента в области порядка комптоновской длины волны мезона и в) более сложные релятивистские эффекты. В этом параграфе мы ограничимся обсуждением порядка величины этих структурных эффектов без проведения количественных вычислений.

Сначала мы обсудим эффект а), рассматривая аномальный магнитный момент $\mu_{\text {ан }}$ нуклеона феноменологически, т. е. считая его пропорциональным вектору из матриц Паули, без учёта происхождения этого момента и его пространственного распределения. Этот момент, конечно, даёт большую, зависящую от спина поправку, ж сверхтонкой структуре. Но, как показано $\mathrm{B}^{19}$, такой момент создаёт ещё небольшой дополнительнй потенциал, не завися̆щий от направления спина. Тогда для атома водорода оператор дополнительной потенциальной энергии $U(\mathbf{r})$, обязанный своим происхождением аномальному моменту протона $\mu_{p}$, ан, равен:

$$
U(\mathbf{r})=4 \pi \mu_{p}, \text { ан }\left(\frac{e^{2}}{4 M_{p}^{2}}\right) \delta(\mathbf{r})
$$

Сдвиг уровня $\langle U\rangle$ за счёт этого потенциала равен нулю для всех состояний ненулевого орбитального момента и $+0,025$ Mat для $2 S$-состояния водорода. Так как аномальные моменты протона и нейтрона почти равны по величине и противоположны по знаку, то соответствующим сдвигом уровня дейтерия можно пренебречь.

Для водорода потенциал, подобный (16), создаётся также дираковской частыю магнитного момента протона. Этот потенциал является дарвиновским членом ${ }^{20,21}$ для протона и даёт поправку 0,006 Maw к сдвигу $2 S$-уровня. Порядок этого члена относительно тонкой структуры равен $\left(\begin{array}{c}m \\ M\end{array}\right)^{2}$ a. Это только один из нескольких до скх пор не вычисленных членов такого, не интересующего нас порядка. Все эти члены мы опустим.

Далее рассмотрим эффект б). Заржженное мезонное облако вокруг нуклеона, повидимому, размазано в области порядка комптоновской длины волны мезона $\lambda$ (около $10^{-13} \mathrm{~cm}$ ). Это размазывание зарлда даёт малый электростатический поправочный потенциал (подобный описанному в $\$ 4)$, который заметен только на расстоянии порядка не более чем $\lambda$. Член Фолди (16) - частичное приближение к этому потенциалу. Компонента Фурье этого потенциала для изменений импульса $q$, малых по сравнению с умноженной на $c$ массой мезона, должна быть практически независимой от $q$ и пропорциональной пространственному объёмному интегралу от потенциала. Это как раз и есть компонента Фурье для малых значений $q$, которая измерялась в экспериментах по электронно-нейтронному взаимодей- 
ствию. Последние экспериментальные данные для пространственного интеграла от этого потенциала дают ${ }^{22}$ :

$$
\left(\frac{4 \pi r_{0}^{3}}{3}\right)(4100 \pm 1000) \boldsymbol{\theta 6}
$$

Хотя потенциал (16) - величина приближённая ${ }^{23}$, всё же его объёмныи интеграл точно совпадает с экспериментальным значением (17). Так как этот потенциал заметно отличен от нуля только в области, много меньшей атомных размеров, соответствующий ему сдвиг уровня зависит только от этого интеграла по объёму, а не от формы потенциальной кривой. Таким образом, величина $0,025 \mathrm{Mzw}$, вычисленная ранее, является достаточно точным приближением.

Если бы существовала надёжная мезонная теория, можно было бы вычислить эффект атомной тонкой структуры типа в), а также б). Такие эффекты возникают за счёт процессов, в которых нуклеон испускает виртуальный мезон, взаимодействующий электромагнитно с атомным электроном и впоследствии снова поглощаемый нуклеоном. Эти эффекты приводят к возникновению аномальных магнитных моментов нуклеонов, а следовательно, к потенциалу (16) и к размазыванию зарлда. Релятивистский расчёт этих эффектов в принципе может дать какие-то изменения по сравнению с приведёнными выше нерелятивистскими оценками, например, существенно изменятся потенциалы, у которых компоненты Фурье пропорциональны положительным степеням импульса $q$ (при $q$ порядка 100 Mas/c). Такие потенциалы не могут наблюдаться в обычных экспериментах по нейтронно-электронному взаимодействио, так как в нём играют роль малые (в ядерной шкале) значения $q$. В атоме, однако, атомные өлектроны имеют хотя и малую, но конечную вероятность обладать очень большими импульсами. Таким образом, возможно, что такие эффекты. от релятивистской структуры дадут заметную поправку к сдвигу уровня, но поправки, превышающие малые доли мегацикла, едва ли возможны.

Похоже на то, что все структурные эффекты (кроме дарвиновского члена, которым мы пренебрегаем) будут противоположного знака и приблизительно равны по величине для нейтрона и протона соответственно. Поэтому мы пренебрегаем влиянием структуры ядра на лэмбовский сдвиг. в дейтерии. В водороде мы получили сдвиг $+\left(0,025+\varepsilon_{s t}\right) M 2$, где $\varepsilon_{s t}$ обозначает дополнительный эффект; упоминаемый в этом параграфе.

\section{§ 6. ВЫводЫ}

Окончательные теоретические величины для лэмбовского сдвига соответственно в водороде и дейтерии равны:

$$
\begin{aligned}
& S_{\mathrm{H}}=\left[1057,19-22 \varepsilon_{\alpha}+S_{Z}^{(3)}+S_{R}-0,04 \varepsilon_{\mu}+\varepsilon_{s t} \pm 0,13\right] M z w_{,},(18 \mathrm{a}) \\
& S_{\mathrm{D}}=\left[1058,49-22 \varepsilon_{\alpha}+S_{Z}^{(3)}+S_{R}-0,02 \varepsilon_{\mu} \pm 0,13\right] M z w_{3},
\end{aligned}
$$


в то время как последние әкспериментальные результаты

$$
\begin{aligned}
& S_{\mathrm{H}}=(1057,77 \pm 0,10) \mathrm{M} 2 \mathrm{u}_{3}, \\
& S_{\mathrm{D}}=(1059,00 \pm 0,10) \mathrm{Mz} \mathrm{s}_{.} .
\end{aligned}
$$

Теоретическое значение для разности между сдвигами уровней в дейтерни и водороде равно:

$$
S_{\mathrm{D}}-S_{\mathrm{H}}=\left(1,296+0,019 \varepsilon_{\mu}-\varepsilon_{s t} \pm 0,035\right) M 2 z^{\prime},
$$

а соответствующее экспериментальное значение

$$
S_{\mathrm{D}}-S_{\mathrm{H}}=(1,23 \pm 0,15) \text { Mazs. }
$$

Таким образом, расхождение между теоретическими и экспериментальными значениями для лэмбовского сдвига как для водорода, так и для дейтерия равно приблизительно половине мегацикла. Существующая неопределённость в $\varepsilon_{\alpha}$ и $\varepsilon_{\mu}$ лишь увеличивает численную ошибку в (18a) и (18b) до $0,16 \mathrm{Mzw}$. Хоропее совпадение межлу теоретическими $(20)$ и экспериментальцыми $(21)$ значениями для $\left(S_{\mathrm{D}}-S_{\mathrm{H}}\right)$ указывает на то, что $\varepsilon_{s t}$ невелико и что это расхождение, повидимому, не связано с эффектами, зависящими от структуры и массы ддер.

Расхождения между теоретическими и экспериментальными величинами для сдвигов втрое превосходят вероятную ошибку и до сих пор не объяснены. Если отбросить возможность экспериментальных и теоретических ошибок, то возможно эти расхождения могут быть объяснены поправками шестого порядка. Эти поправки могут иметь величину порядка $1 / 2$ Mәш в том случае, если при некоторых членах будут стоять неожиданно большие численные коэффициенты. Точное измерение лэмбовского сдвига для однажды ионизованного гелия может пролить некоторый свет на зависимость от заряда ядра некоторых из этих членов.

\section{\$7. ДОПОЛНЕНИЕ}

\begin{tabular}{|c|c|c|c|}
\hline $\begin{array}{l}\text { Пара- } \\
\text { граф }\end{array}$ & $\begin{array}{l}\text { Сим- } \\
\text { волI }\end{array}$ & Значение & $\begin{array}{c}\text { Вклад в лэмбовский } \\
\text { сдвиг }\end{array}$ \\
\hline 2 & $S_{\infty}^{(1)}$ & $\begin{array}{l}\text { Все радиационные поправки вто- } \\
\text { рого порядка для бесконечно тяжё- } \\
\text { лого ядра . . . . . . . . . . . }\end{array}$ & $(1052,14 \pm 0,08)$ M2楼 \\
\hline 2 & $\varepsilon_{\alpha}$ & $\begin{array}{l}\text { Ошнбка за сqёт неопределённости в } \\
\text { qисленном значения константы тон- } \\
\text { кой структуры. }\end{array}$ & $(+0,05) \mathrm{Ma}$ \\
\hline 2 & $S(2)$ & Все поправки четвёртого порядка & $-(6,20 \pm 0,10) \mathrm{MaL}$ \\
\hline
\end{tabular}

Для удобства читателя даём ниже короткую сводку различных членов, обсуждённых в этой статье, с указанием параграфов, в которых они рассмотрены, и вносимого ими вклада в лэмбовский сдвиг. 


\begin{tabular}{|c|c|c|c|}
\hline $\begin{array}{l}\text { Пара- } \\
\text { граф̆ }\end{array}$ & $\begin{array}{c}\text { Сим- } \\
\text { вол }\end{array}$ & Знатение & $\begin{array}{c}\text { Вклад в лэмбовский } \\
\text { сдвиг }\end{array}$ \\
\hline 2 & $S_{Z}^{(3)}$ & $\begin{array}{l}\text { Поправки шестого порядка и выс- } \\
\text { шей степени по } Z \text {. . . . . . }\end{array}$ & Неизвестен \\
\hline 2 & $S_{\mathrm{R}}$ & $\begin{array}{l}\text { Все остальные радиационные по- } \\
\text { правки для бесконечно тяжёлого ядра }\end{array}$ & Неизвестен \\
\hline 3 & $\Delta S_{\mathrm{H}}$ & $\begin{array}{l}\text { Влияние конечной массы ядра на } \\
\text { сдвиг уровня водорода . . . . . }\end{array}$ & $-(1,175 \pm 0,08) M 21$ \\
\hline 3 & $\varepsilon_{p}$ & $\begin{array}{l}\text { Ошибка за счёт аномального маг- } \\
\text { нитного момента электрона в множи- } \\
\text { теле, учитывающем поправки за счёт } \\
\text { конечной массы ядра } . . . \\
.\end{array}$ & $\begin{array}{l}+0,08 M 2 u_{4}(\mathrm{H}) \\
+0,04 M 2 u_{3}(\mathrm{D})\end{array}$ \\
\hline 4 & $\Delta V$ & Учёт конечного радиуса дейтерона & $(0,733 \pm 0,025) M 2 t$ \\
\hline 5 & $U$ & $\begin{array}{c}\text { Уqёт электронно-нуклеонного } \\
\text { взаимодействия для водорода . . . }\end{array}$ & $(0,025 \pm 0,005) \mathrm{Mzu}$ \\
\hline 5 & $\varepsilon_{s t}$ & $\begin{array}{l}\text { Дополнительные эффекты, обуслов- } \\
\text { ленные структурой ядра.. . . . . }\end{array}$ & Неизвестен \\
\hline
\end{tabular}

ЦИТИРОВАННАЯ ЛИТЕРАТУРА

1. W. E. L a mb and R. C. Retherford, Phys. Rev. 79, 549 (1950), 81, 222 (1951); 86, 1014 (1952).

2. W. E. L a m b, Phys. Rev. 85, 259 (1952).

3. Triebwasser, Dayhoff and Lamb, Phys. Rev. 89, 98 (1953).

4. Bethe, Brown a. Stehn, Phys. Rev. 77, 370 (1950).

5. H. A. B e th e, Phys. Rev. 72, 339 (1947).

6. N. M. Kroll a. W. E. L a m b, Phys. Rev. 75, 388 (1949); J. B. French and V. F. We is sk opf, Phys. Rev. 75, 1240 (1949); J. Schwinger, Phys. Rev. 76, 790 (1949).

7. R. P. Fe y n m a n, Phys. Rev. 76, 769 (1949).

8. Day hoff, Triebwasser a. La mb, Phys. Rev. 89, 106 (1953).

9. R. Karplus a. A. Kle in, Phys. Rev. 85, 972 (1952); N. M. Krol1 and F. P o $110 \mathrm{ck}$, Phys. Rev. 86, 876 (1952); E. E. S al pet er and W. A. N e w c o m b, Phys. Rev. 87, 150 (1952).

10. J. W. M. Du Mond and E. R. Cohen, Phys. Rev. 82, 555 (1951).

11. M. Baranger, Phys. Rev. 84, 866 (1951); Karplus, K1ein, and S c h w ing er, Phys. Rev. 86, 288 (1952).

12. R. Karplus and N. M. Kroll, Phys. Rev. 77, 536 (1950).

13. Baranger, Dyson a. Salpeter, Phys. Rev. 88, 680 (1952).

14. Bers ohn, We neser a. Krol1, Phys. Rev. 86, 596 (1952).

15. E. E. S a 1 p et e r, Phys. Rev. 87, 328 (1952).

16. J. Breit and G. E. Brown, Phys. Rev. 74, 1278 (1949).

17. J. M. B latt a. J.D. J a ck s o n, Phys. Rev. 76, 18 (1949); H. A. B e the, Phys. Rev. 76, 38 (1949); E. E. S al peter, Phys. Rev. 82, 60 $(1951)$.

18. F. L o w, Phys. Rev. 77, 361 (1950).

19. L. L. Fold y, Phys. Rev. 83, 688 (1951). 
20. C. G. D a r w in, Proc. Roy. Soc. (London) A118, 654 (1928). 21. L. L. Foldy and S. A. Wo ut hu ysen, Phys. Rev. 78, 29 (1950).

22. Hamermesh, Ringo a. Wat te nberg, Phys. Rev, 85, 483 (1952). 23. B. D. Frie d, Phys. Rev. 86, 434 (1952); S. B or o w it $z$, Phys. Rev. 86, 567 (1952).

Дополнительный список литературы

на русском языке по вопросам лэмбовскогосдвига и других радиационных поправок

1. В. $\Phi$. В а й с к о п $\Phi, У Ф Н ~ 41,165$ (1950).

2. Я. А. С м о р о ди и н к и й, УФН 39,325 (1949).

3. Научно-реферативные сборники «Пробламы современной физики", выпуск 6, 1948 г.; выпуск 1, 1950 г.; выпуск 11, 1951 г.

4. Сдвиг уровней атомных электронов. Сборник, ИЛ, Москва, 1950 г.

5. А. А. С око ло в и Д. Д. Ив ан е н ко, Квантовая теория поля. Гостехиздат, Москва, 1952 r.

6. У. Е. Лэм 6 и Р. К. Ри з ерфорд, Тонкая структура водородного атома, У $\Phi H ~ 45,553$ (1951). 